\title{
A Comparison between Orion Automated and Space Shuttle Rendezvous Techniques
}

\author{
Jose P. Ruiz and Jeremy Hart \\ NASA Johnson Space Center, Houston, TX 77058
}

Extended Abstract

The Orion spacecraft will replace the space shuttle and will be the first human spacecraft since the Apollo program to leave low earth orbit. This vehicle will serve as the cornerstone of a complete space transportation system with a myriad of mission requirements necessitating rendezvous to multiple vehicles in earth orbit, around the moon and eventually beyond. These goals will require a complex and robust vehicle that is, significantly different from both the space shuttle and the command module of the Apollo program. Historically, orbit operations have been accomplished with heavy reliance on ground support and manual crew reconfiguration and monitoring. One major difference with Orion is that automation will be incorporated as a key element of the man-vehicle system. The automated system will consist of software devoted to transitioning between events based on a master timeline. This effectively adds a layer of high level sequencing that moves control of the vehicle from one phase to the next. This type of automated control is not entirely new to spacecraft since the shuttle uses a version of this during ascent and entry operations. During shuttle orbit operations however many of the software modes and hardware switches must be manually configured through the use of printed procedures and instructions voiced from the ground. The goal of the automation scheme on Orion is to extend high level automation to all flight phases. The move towards automation represents a large shift from current space shuttle operations, and so these new systems will be adopted gradually via various safeguards. These include features such as authority-to-proceed, manual down modes, and functional inhibits. This paper describes the contrast between the manual and ground approach of the space shuttle and the proposed automation of the Orion vehicle. I will introduce typical orbit operations that are common to all rendezvous missions and go on to describe the current Orion automation architecture and contrast it with shuttle rendezvous techniques and circumstances.

The shuttle rendezvous profile is timed to take approximately 3 days from orbit insertion to docking at the International Space Station (ISS). This process can be divided into 3 phases: far-field, mid-field and proximity operations. The far-field stage is characterized as the most quiescent phase. The spacecraft is usually too far to navigate using relative sensors and uses the Inertial Measurement Units (IMU's) to numerically solve for its position. The maneuvers are infrequent, roughly twice per day, and are larger than other burns in the profile. The shuttle uses this opportunity to take extensive ground based radar updates and keep high fidelity orbit states on the ground. This state is then periodically uplinked to the shuttle computers. The targeting solutions for burn maneuvers are also computed on the ground and uplinked. During the burn the crew is responsible for setting the shuttle attitude and configuring the propulsion system for ignition. Again this entire process is manually driven by both crew and ground activity. The only automatic processes that occur are associated with the real-time execution of the burn. The Orion automated functionality will seek to relieve the workload of both the crew and ground during this phase. The Orion will be equipped with GPS enhancing its onboard state accuracy and 
maneuver computations will be put onboard as well. The automation is further enhanced with the highlevel sequencing functionality that will toggle between distinct coast and burn software segments.

The mid-field rendezvous phase starts as the spacecraft utilizes relative sensors for onboard navigation. The shuttle approaches this phase with a fairly limited suite of onboard tools. The crew configures software to begin taking relative measurements and monitors the data as it is incorporated. The crew also manually initiates targeting routines onboard the shuttle. All of this activity is still coordinated through pre-printed timelines that are anchored to various mission events. This timeline management is complicated even further if malfunctions occur and other procedures must be inserted. The level of activity and communication with the ground increases and typically 4 astronauts are dedicated to the various tasks. The Orion will have the same onboard functionality elements such as onboard navigation and targeting but will have the high level sequencer active as well. This sequencer will operate based on the preloaded timeline and will serve to initiate many activities that the astronauts had to once manually enter. For instance, manually cueing up attitude maneuvers and targeting routines will be automatically triggered off of the timeline. Critical events such as burns however will typically have an authority to proceed (ATP) or a pause in the computer program that awaits a crew input. The added functionality will aid the crew in the very different environment of the Orion. The Orion flight deck will be constrained and only have 2 fully suited crew opposed to the relatively spacious and shirtsleeve environment of the shuttle.

The final phase of rendezvous is proximity operations. This phase involves the frequent use of jet firings to control the relative trajectory of the vehicle up to docking. The shuttle approaches this by utilizing fully manual control of translation. The commander uses a docking camera and relative sensors to decide the time and magnitude of different burns along the trajectory. The attitude control however is still relegated to the computer. The trajectory control sensor (TCS) is a laser than provides high fidelity relative measurements hosted on a laptop mounted on the flight deck. Another crew member is charged with maintaining these laptops views and arranging the information for the commander's situational awareness and piloting. Another crew member voices procedures of the printed timeline and other cues for situational awareness. Yet another crew member aims a hand held laser out the window at the ISS for back up relative navigation data. This phase consists of the highly trained and tightly choreographed interplay between the $4 \mathrm{crew}$ members. The entire process is autonomous because the crew has become much less dependent on the ground by this time. It still requires months of training and simulation and is very crew intensive. The Orion will help alleviate some of this by actively flying the vehicle in proximity operations. The relative guidance system will be tied into the sensor data and jet system to give the computer control of the relative trajectory. Of course there will be on option to manually override this mode in case of malfunctions. Furthermore, the high level sequencer will transition from one mode to the next as the vehicle makes its way to the ISS. The two person Orion crew will have access to graphical views of the approach incorporating the onboard sensors for heightened situational awareness as well.

The planned differences between the Orion and space shuttle are quite extensive. Some of these differences are subtle and others are a large shift. The key to the automation approach is the high level sequencer and it's use and flexibility during real-time operations. 